Folia phoniat. 1973;25:1-8

\title{
Zum 80. Geburtstag von Prof. Dr. Miloslav Seeman
}

\begin{tabular}{|l|l|l|}
\hline E. & & Sedláček \\
\hline K. & & Sedláček \\
\hline
\end{tabular}

\section{Prag}

Am 15. Februar 1972 feierte Prof. Dr. Miloslav Seeman, eine der hervor-ragendsten Persönlichkeiten der heutigen phoniatrischen Welt, der Begründer der Phoniatrie in der Tschechoslowakei, Mitbegründer und Redakteur der Folia Phoniatrica, in voller geistiger und körperlicher Frische an seiner phoniatrischen Klinik der Prager Karls-Universität seinen 80. Geburtstag.

Prof. Seeman ist im Jahre 1892 in Vysoké Veselí, einer kleínen Stadt in Ostböhmen. geboren. In seinem siebenten Lebensjahr übersiedelten seine Eltern nach Wien, wo sein Vater als Beamter angestellt war. Das Studium der Medizin absolvierte Seeman in Wien. Schon als Mediziner arbeitete er im anatomischen Institut von Prof. Hochstetter und dann an der I. Chirur-

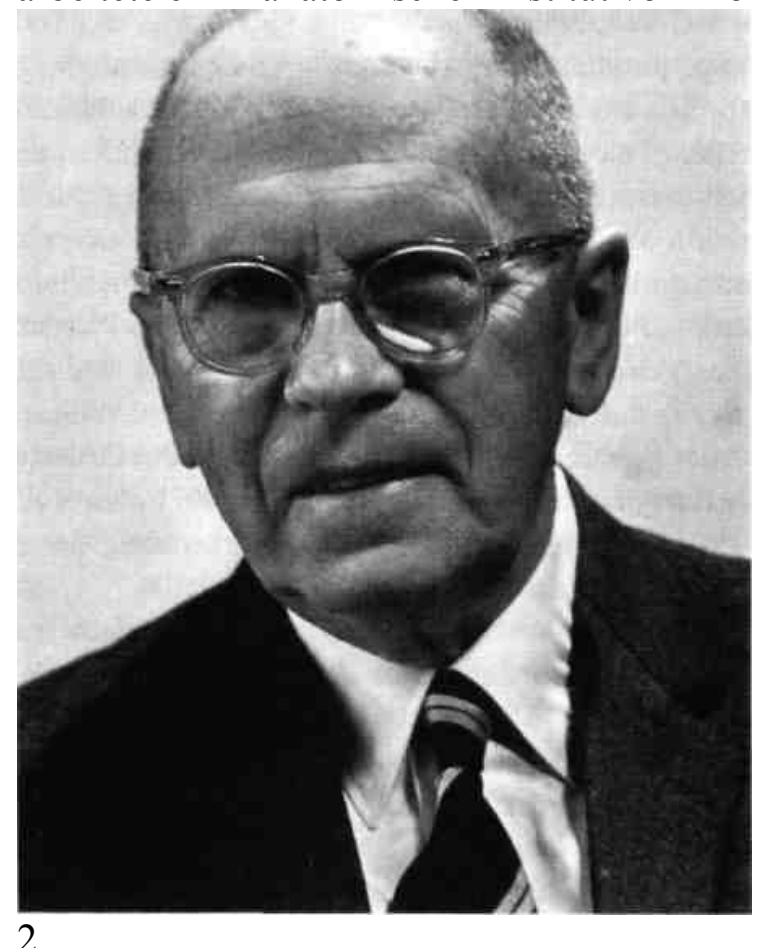

Sedlácek/Sedlácek

gischen Universitätsklinik von Prof, von Eiselsberg. Aus dieser Zeit stammt auch seine erste Arbeit [1]. Nach der Promotion (1917) wechselte er an die otolaryngologische Klinik von Prof, von Chiari und dann von Prof. Hajek. Er hat sich sehr bald entschlossen, sich der Sprach- und Stimmheilkunde zu widmen und hat deshalb von 1918 bis 1919 in Berlin im Ambulatorium für 
Sprach- und Stimmheilkunde (welches einen Teil der HNO-Klinik Prof. Kilians bildete) bei Prof. Gutzmann, dem Begründer dieses medizinischen Faches, gearbeitet. Gleichzeitig hatte er auch die Möglichkeit, Schäffers Laboratorium für Akustik an der otologischen Klinik von Prof. Passow und die Abteilung für Hördefekte und Sprachstörungen von Prof. Flattau zu besuchen. $1 \mathrm{~m}$ Frühling 1919 kehrte er nach Wien zurück und begründete an der dortigen laryngologischen Universitätsklinik ein phonetisches Laboratorium und eine Stimm- und Sprachstörungsambulanz, die später Prof. Stern und dann Prof. Trojan leiteten. Sie existieren noch heute und werden von Dr. Frank geführt.

Nach der Proklamation der Selbständigkeit des tschechoslowakischen Staates kehrte Prof. Seeman in seine Heimat zurück. 1m Jahre 1921 wurde er nach Prag berufen und arbeitete an der HNO-Universitätsklinik von Prof. Kutvirt, der viel Verständnis für das neue Fach hatte, so dass Seeman dort eine selbständige phoniatrische Abteilung gründen konnte mit dem Namen «Abteilung für Stimm- und Sprachstörungen und Hördefekte ». So entstand die erste Abteilung, die alle Komponenten des Kommunikationsprozesses in ihrer logischen Verbindung zusammenbrachte, was der Konzeption ent-sprach, die schon am Anfang seiner Arbeit Prof. Seeman prägte. Er schuf auch den Namen dieses Faches gemäss der Analogie Psychologie Psych-iatrie: Phonetik - Phoniatrie, das ist die medizinische Phonetik. Das Ein-schliessen eines gewissen Teiles der Pathophysiologie des Gehörs, insbe-sondere mit Bezug auf Pathologie der Sprache und Rehabilitation der Hördefekte (nicht der ganzen Audiologie), wurde auch vom Gründungskongress der Union der Europäíschen Phoniater im Herbst 1971 als absolut notwendig anerkannt, was gleichzeitig auch die voile Anerkennung der SEEMANschen Konzeption der Phoniatrie ist.

Im Jahre 1923 unternahm Seeman eine Studienreise nach Frankreich, wo er bei Prof. Rousselot am College de France und bei Prof. Poirot an der Sorbonne arbeitete.

Der Beginn der Phoniatrie in Prag war anfangs sehr bescheiden: es be-stand ein einziger Raum, der als Ambulatorium und phonetisches Laboratorium diente. Von hier aus begann Seeman systematisch, dieses neue Fach in seiner Heimat aufzubauen. Man kann seine Arbeit in vier Etappen auf-

Zum 80. Geburtstag von Prof. Dr. Miloslav Seeman

3

teilen, obwohl sich alle Etappen untereinander verbanden. Die erste war die Gründung und Verbreiterung der wissenschaftlichen Basis des neuen Faches und die Verbreitung der Kenntnisse aus diesem Fache in ärztlichen und pädagogischen Kreisen. Die zweite Etappe war der Aufbau einer eigenen wissenschaftlichen Schule und die Ausbildung weiterer Ärzte sowie die Er-richtung von phoniatrischen Abteilungen an alien HNO-Universitätskliniken in der Tschechoslowakei. Zur dritten Etappe gehört die Eingliederung der Phoniatrie in die Organisation des Gesundheitswesens. Und die Endetappe stellt die Errichtung phoniatrischer Bettenabteilungen in grossen medizini-schen Zentren des Staates dar. Dieser planmässige Übergang von der indi-viduellen, hervorragenden Arbeit eines exklusiven wissenschaftlichen For-schers zu einer breiten Aufbau- und Organisationstätigkeit ist eines der grössten Verdienste von Seeman urn die tschechoslowakische Phoniatrie. Denn für die Existenz eines Faches genügen die Kenntnisse einiger hervor-ragender Wissenschaftler allein nicht; diese Kenntnisse müssen von vielen Fachleuten realisiert und in breiter medizinischer Praxis ausgenützt werden. In der Tschechoslowakei gibt es jetzt neben der Prager phoniatrischen Universitätsklinik, die als Ausbildungsstätte für Phoniatrie dient, 7 phoniatrische Abteilungen an den HNO-Kliniken und 
viele phoniatrische Abteilungen, an denen insgesamt 52 Spezialärzte für Phoniatrie arbeiten; von diesen sind 2 Professoren und 2 Dozenten der Phoniatrie.

Prof. Seeman habilitierte sich im Jahre 1925 für das Fach der Phoniatrie, wurde 1931 zum ausserordentlichen und 1939 zum ordentlichen Professor der Phoniatrie ernannt. An der phoniatrischen Klinik in Prag hat Prof. Seeman auch ein selb-ständiges wissenschaftliches phoniatrisches Forschungslaboratorium der Fakultät der allgemeinen Medizin der Karls-Universität errichtet, als dessen Direktor er bis heute tätig ist.

Prof. Seeman organisierte eine Reihe von systematischen präventiven Aktionen. Vor allem war es seit 1929 eine systematische Aufsuchung und Behandlung von Sprach- und Stimmstörungen und Hördefekten in den Kindergarten; er veranlasste periodische präventive Untersuchungen der Schüler von Staatskonservatorien und anderen Gesangsschulen, Vorlesungen über die Hygiene der Stimme an diesen Schulen sowie systematische Untersuchungen des Gehörs von Neugeborenen und Kindern im Vorschulpflichts-alter, so dass die phoniatrische Betreuung auf das Gebiet des ganzen Staates verbreitet wurde.

Die wissenschaftliche Arbeit Prof. Seemans enthält nach beigefügtem Verzeichnis 121 Arbeiten, von denen viele für die Phoniatrie eine erstrangige

4

Sedlácek/Sedlácek

Bedeutung haben. Seeman führte als erster die Stroboskopie in die Stimm-pathologie in seinen Arbeiten über die laryngoskopische Untersuchung und Behandlung bei einseitigen

Stimmbandlähmungen ein, welche auch einzig-artige Beobachtungen vieler pathophysiologischer Mechanismen und zu-gleich auch die Prinzipien der Reedukation der Stimme enthalten. Er be-schrieb z. B. die Tatsache, dass bei der Phonation die gelähmte Stimmlippe vertikal, die gesunde hingegen horizontal schwingt. Weiters erklärte er die Entstehung der Diplophonie bei Rekurrenslähmung.

Seeman beschrieb den Mechanismus der Ösophagusstimme und arbeitete die Rehabilitationsmethode zu ihrer Benützung mit Hilfe der Aspiration der Luft in die Speiseröhre aus.

Wir dürfen die Arbeiten nicht vergessen, die sich mit der Etiopathogenese und Therapie des Stotterns und Polterns befassen. Seeman ist der Ansicht, dass das Stottern durch dynamische Abweichungen in der Funktion des Striopallidärsystems entsteht, die emotionell durch Abweichungen der Funktion des vegetativen Nervensystems ausgelöst werden.

Bekannt sind auch die Arbeiten, die sich mit der konstitutionellen Ver-spätung der Sprachentwicklung des Kindes befassen - die Seeman durch eine Dysfunktion der Zerebellovestibularfunktion erklärt -, und die Arbeiten, die sich mit der Beschreibung der Stimm- und Sprachstörungen bei Gaumen-spalte beschäftigen und die phonetischen und chirurgischen Behandlungs-methoden angeben.

Bekannt ist auch das SEEMANsche Symptom bei Myasthenia gravis pseudoparalytica, seine Beschreibung der Falsettstimme und der Sprache und Stimme bei den extrapyramidalen Erkrankungen.

Am meisten verbreitet ist seine Monographic «Die Störungen der Kin-dersprache», die in fünf Sprachen übersetzt wurde und nicht nur seine Le-benserfahrungen enthält, sondern auch eine Reihe von originellen Beíträgen sowohl auf dem Gebiete der Diagnostik als auch der Therapie, und die Grundregeln und Gesetze, welche bei der Rehabilitation der Sprache gelten, angibt. 
Prof. Seeman beteiligte sich an der Begründung der Internationalen Gesellschaft für Logopädie und Phoniatrie (1924), deren langjähriger Vizepräsi-dent er war. 1m Jahre 1930 veranstaltete er den 4. Internationalen Phoniatri-schen Kongress dieser Gesellschaft in Prag.

Prof. Seeman hat zahlreiche Ehrungen erhalten. Er ist Ehrenmitglied der Internationalen Gesellschaft für Logopädie und Phoniatrie (IALP), der Tschechoslowakischen Medizinischen Gesellschaft J. E. Purkyne, der Pol-nischen Otolaryngologischen Gesellschaft, der Ungarischen Gesellschaft für

Zum 80. Geburtstag von Prof. Dr. Miloslav Seeman

5

Phonetik und Phoniatrie, der Deutschen Gesellschaft für Stimm- und Sprach-heilkunde, und er ist Träger vieler ausländischer Orden, des tschechoslowakischen Arbeitsordens und Laureat des tschechoslowakischen Staatspreises. Seine Schüler in der Tschechoslowakei und seine Freunde in der ganzen Welt sowie die Redaktion der Folia Phoniatrica beglückwünschen Herrn Prof. Seeman zu seinem Lebenswerk und wünschen ihm viel Gesundheit und Er-folg in seiner weiteren wissenschaftlichen und pädagogíschen Arbeit.

Eva und Karel Sedlácek

Arbeiten von Prof. Dr. Miloslav Seeman

Zur Nachbehandlungder Amputationsstümpfe. Wien. klin. Wschr. 28; Nr. 13 (1915).

Die phonetische Behandlung bei einseitiger Rekurrenslähmung. Arch. Laryng. Rhinol. 32: Heft 2.

Laryngostroboskopische Untersuchungen bei einseitiger Rekurrenslähmung. Mschr. Ohrenheilk. Laryng. Rhinol. 55: (1921).

Rec a hlas bez hrtanu a její vznik. Cas. Lék. ces. 17 (1922).

O pfícinách nemluvnosti ve vëku dëtském. Cas. Lék. ces. 21 (1922).

Principy foniatrického lécení po uranoplastice. Cas. Lék. ces. 35 (1922).

O tvoreni rejstfíkü hlasových. Hudební výchova (Praha 1923).

O persistujícím hlase fistulovém: pfíspëvek $\mathrm{k}$ pathogenese této poruchy hlasové. Cas. Lék. ces. 4(1923).

O submukosním rozstëpu tvrdého patra. Cas. Lék. ces. 24 (1923).

K. pathogenese opozdëného vývoje feci. Pfíspëvek k otázce o vlivu nozecku na poruchy feci.

Cas. Lék. ces. 51 (1923).

Contribution à la pathogénie et la pathologie de la fissure sousmuqueuse du palais osseux. Arch. int. Laryng. Otol. Rhinol. Broncho-æsophagoscopie, Paris (1924).

O koktavosti a její lécbë. Prakt. lékaf 2 (1924).

O dýchacích a fonacních pohybech oesofagu a jejich grafické registraci. Cas. Lék. ces. 20(1924).

O optické a akustické percepci feci. Cas. Lék. ces. 42 (1924).

Seeman, M. a Smilauerová, L.: Transoesofageální registrace pohybû srdecních. Cas. Lék. ces. 52(1924).

Experimentální a fysiologické studie ke vzniku feci bez hrtanu a zvlást" feci oeso-fageální. Cas. Lék. ces. 26-27 (1924).

Fysiologické základy reedukace sluchu. Cas. Lék. ces. 28 (1925).

Reedukace sluchu pfi hluchonëmosti. Rev. pro vzdël. a výchovu hluchonëmých. No. 9-10(1925).

Phoniatrische Bemerkungen zur Laryngektomie. Arch. klin. Chir. 140: (1926).

Vývoj feîi u dítëte normálního a hluchonëmého. Rev. pro vzdël. a výchovu hluchonëmých. No. 6-7 (1926). 
21 Prodlouzení patra retroposicí horizontálních plotének ossis palatini. Zpráva o sjezdu Cs. otolaryng. spol. (1926); Grégr (1927).

6

Sedlácek/Sedlácek

O úkolech a klinickém významu foniatrie. Cas. Lék. ces. 13-14 (1927).

Význam poruchy feci a grafické registrace funkce vela pro vcasnou diagnosu my-asthenie. Cas.

Lék. ces. 13-14 (1927).

Príciny opozdëného vývoje reci. Prakt. lék. I (1927).

Registrierung ösophagealer Phonationsbewegungen. Vox H. 9 (1927).

O familiárním opozdëní vúvoje reci. Cas. Lék. ces. 18 (1928).

Rec dítëte. Knihovny nových skol. Sv. 2 (1928) (Matei1/8ká skola).

Die Bedeutung der Sprachstörung und der graphischen Registrierung der Funktion des weichen

Gaumens für die frühzeitige Diagnose der Myasthenie. Otolaryng. slav. /: (1929).

Profesor MUDr. Otokar Kutvirt. Prakt. lékaf 15 (1929).

Profesor MUDr. Otokar Kutvirt. Mschr. Ohrenheilk. 8-9 (1929).

Sur le développement retardé de la parole se présentant héréditairement dans la famille.

Otolaryng. slav. 2: (1930).

Pohlavní funkce a hlas. Cas. Lék. ces. 2 (1930).

Zur Physiologie der Oesophagusperistaltik. Arch. klin. Chir. 160: (1930).

Les functions sexuelles et la voix. Otolaryng. slav. 2: (1930).

Gehör und Sprache. Wien. med. Wschr. 35 (1930).

K. registraci polykacích pohybû. Cas. Lék. ces. 13 (1930).

O pfícinách a lécení hluchonëmosti. Ochylná mládez (1930).

O zkusenosti s velofaryngografií z hlediska foniatrického. Cas. Lék. ces. 47 (1931).

Zur Pathologie des tschechischen f (f bilabiale). Otolaryng. slav. 3: (1931).

Vývoj reci a jejíporuchy ve vëku dëtském. Vých. cít. (1931).

K etiologii koktavosti. Zemanûv sbornik (1931).

O nedoslýchavosti. Cas. Cerv. kfíze (1931).

Sur Timportance acoustique des cavités sous-glottiques. Cas. pro moderní filologii 16: 12(1931).

Androglottie bei Schwestern. Wien. med. Wschr. 28-30 (1932).

Über somatische Befunde bei Stotterern. Mschr. Ohrenheilk. 8 (1934).

Nëkolik úvah o methodë globálního ctení. Prakt. lék. 10 (1934).

Význam pathologie dvojcat pro studium chorob reci. Cas. Lék. ces. 44-45 (1935).

O podstatë koktavosti. Slovo a slovesnost 2 (1935).

Piíspëvek k pathogenese koktavosti. Rev. pro neurologii a psych. 5-6 (1936).

Hesla z foniatrie. Kalendáf Lék. ces. (1937).

Die Bedeutung der Zwillingspathologie für die Erforschung von Sprachleiden. Arch. SprachStimmheilk. I: 2 (1937).

Prevence vad sluchu, chorab reci a hlasu skolní mládeze. Cas. Lék. ces. 10 (1938).

O poruchách reci. Spirála (1938).

16 hesel z foniatrie v Pedagogické Encyklopedii. Nakl. Novin (1938-1940).

O úcasti a poslání lékafe v péci o hluchonëmé. Sbornik k ctvrtstoleti Spolku pro péci o

hluchonëmé, 1915-1940. R. (1940).

Pfipomínky foniatra ke zpëvu v pfedskolním vëku. Hudba a skola (1940). 
Obdobi mutace a hlasová výchova. Hudba a skola (1940).

O zdravotním významu zpëvu. Zdraví lidu (Mës. Cerv. kfíze) 3 (1940).

Fonetická a foniatrická pozorování u rozstëpu patra. Cas. Lék. ces. 80: 1413 (1941).

Zum 80. Geburtstag von Prof. Dr. Miloslav Seeman

7

Untersuchungen über Phonationsdauer bei Stotterern. Arch. Sprach-Stimmheilk. 5: Heft 2(1941).

O nëkterých zvlástních zpûsobech tvofení hiasu. Slovo a slovesnost VII ses. 2 (1941).

Vývoj a poruchy dëtské feci. St'astné dítë (1941).

O mutismu. Gas. Lék. ces. 81: 1353 (1942).

Cyklické promëny lidského hiasu. Vëda a zivot (1943).

Závislost fonacní doby na neurovegetativní regulaci. Cas. Lék. ces. 83: 714 (1944).

Kritické poznámky k stroboskopii hrtanu a návrh na její zdokonalení novým elektro-akusticky rízeným mikrofon-stroboskopem. Cas. Lék. ces. 84: 1452 (1945).

Péce o fee a hlas na matefských skolách. Nova skola /: No. $5(1945 / 46)$.

Péce o dëtský hlas v pfedskolní dobë. Pfedskolní výchova. No. 1-2 (1946).

O medikamentosní lécbë pri koktavosti. Cas. Lék. ces. 85: 1128 (1946).

Hlas a vnitfní sekrece. Skalpel No. 3-4 (1947).

Seeman, M. et Tarneaud, J.: La voix et la parole (Paris 1950).

Sur la regulation neurovégétatíve de la durée de la phonation. Folia phoniat. /: (1948).

Poznámky foniatra k uranoplastice. Cas. Lék. ces. (1950).

Organizace foniatrické péce v CSR. Cas. Lék. ces. 89: 1151 (1950).

Ükoly foniatra pri lécbë rozstëpu patra. Cas. Lék. ces. 89: 407 (1950).

Nouvelles theories sur la pathogénie du bégaiement. Presse méd. No. 9 (1951).

Metodika rehabilitace hiasu po larynektomii. Cas. Lék. ces. 90: 1359 (1951).

Novoe poglady u pathogenesie jakania. Otolar. Polska Nr. 1 (1952).

Kapitoly v Repetitoriu prakt. lékafe: Poruchy feci. Hluchonëmost. Mutace (St. zdrav. nakl., Praha 1952).

Kapitoly v ucebnici Otolaryngologie: Fysiologie hiasu. Hluchonëmost. Rhinophonie (St. zdrav. nakl., Praha 1953).

O lidském hiasu. Nakl. Csl. spolecnosti pro sífení polit. a vëdeckých znalostí, p. 53 (Orbis, Praha 1953).

Poruchy dëtské feci, p. 265 (St. zdrav. nakl, Praha 1955).

Prevence poruch feci v dëtském vëku. Rodina a skola. Min. skolství (1956/II).

O neurosách feci. Csl. Cerv. Kfíz, No. 3 (1956).

Hlasová hygiena v období mutace. Csl. Cerv. Kfíz, No. 9 (1956).

Symptômes extrapyramidaux de la voix et de la parole. Proc. 10th Int. Speech Voice Therapy

Conf., Barcelona 1957. Acta oto-rino-laring. ibero-amer. (1957).

O vývoji foniatrie. Csl. Otolaryng. VI/5: 257 (1957).

Zur Pathologie der Oesophagus-Stimme. Folia phoniat. 10: 44 (1958).

Sprachstörungen bei Kindern, p. 392 (Marhold, Halle/Saale 1959).

Rehabilitace osob po operaci zhoubných nádorû hornich cest dýchacich a zvlástë hrtanu. Sborník mezinárodního sjezdu ORL, Brno (1959).

Reabilitacao da voz e da fala após a laringectomia. Atualides méd. biol., Rio de Janeiro Nr. 19 (1959).

Neurologie und Sprachheilkunde. Aktuelle Probleme der Phoniatrie und Logopädie. Folia phoniat. Suppl. /: 127-133 (1960). 
Historisches zur Entstehung der Phoniatrie. Sprachforum 1960.

Ceská slovní audiometrie. Seeman a kolektiv (St. zdrav. nakl., Praha I960).

Nouveaux résultats des recherches expérimentales dans la reeducation de la voix æsophagienne.

J. franc. Oto-Rhino-Laryng. 10: 47-56 (1961).

8

Sedlácek/Sedlácek

Die Bedeutung des Oesophagusmundes für die Rehabilitation der Stimme nach Laryngectomie.

Kongr.Ber. Gemeinschaftstag. allg. Phonetik, Hamburg 1960.

Rehabilitacja glosu i mowy po wycieciu krtani. Logopedia, Lublin, No. 2 (1961).

Hlasová hygiena v období mutace. Cas. Cerv. Kfíze, No. 8 (1962).

Rasstrojstva reïi v dëtskom vozrastë, p. 298 (Medgiz, Moskau 1962).

Predmluva k obrázkovým testûm pro vysetrování feci M. Truhlárové (St. zdrav. nakl., Praha 1962).

Predmluva: Sedlácek Sychra Hudba a slovo z exper. hlediska. (St. hud. vydav., Praha 1962).

Über die Früherfassung und Betreuung hörgeschädigter Kinder in der Tschecho-slowakei.

Mschr. Ohrenheilk. Laryng. Rhinol., 96: (1962).

Über die Motorik bei Polterern; in Seeman and Novak Proc. 12th Int. Speech Voice Therapy

Conf., Padua 1962.

Ein Beitrag zum elektíven Mutismus. Wiss. Z. Univ. Halle, pp. 1675-1682 (1962).

Über die Motorik bei Polterern; in Seeman und Novak. Folia phoniat. 15: 170-176 (1963).

Predmluva: J. Kiml. Neurodynamické poruchy hlasu (St. zdrav. nakl., Praha 1963).

Klanganalyse und Hòrprüfung; in Seeman und Petrík. Z. Phonetik, Sprachwiss.

Kommunikationsforsch. 16: 201-209 (1963).

7 hesel do: Enzyklopädisches Handbuch der Sonderpädagogie und ihrer Grenzgebiete (Marhold, Berlin 1965) - 6. (Hesla jsou zpracována velmi podrobnë).

Zur Diagnostik der Suprabulbären-Dysarthrie. Mschr. Ohrenheilk. Laryng. Rhinol. 99: 78-82 (1965).

Sprachstörungen bei Kindern; 2. Aufl., p. 440 (VEB Verlag Volk und Gesundheit, Berlin 1965).

LEtiopathogenèse du bégaiment et du bredouillement. Rev. Laryng. Otol. Rhinol. 86: 1009-1020

(1965).

Statische Störungen und Sprachentwicklung. De therapia vocis et loquelae, 13. Congr., Wien 1965.

Akademik Pfecechtël jako clovëk a vëdec - Pfílezitostný svazek k 80. narozeninám (Knihtisk n.p., Praha 1965).

Speech pathology in Czechoslovakia; in Speech pathology, pp. 439-457 (North-Holland, Amsterdam 1966).

Les troubles du langage chez Гenfant, p. 312 (Presses Académiques, Bruxclles/ Maloine, Paris 1967).

Rehabilitation of laryngectomized subjects. Acta oto-laryng., Stockh. 64: 235-242 (1967).

Tal Rubbningar hosbarn, p. 219 (Fritzes, Stockholm 1968).

La reeducation et la rehabilitation des troubles du langage, de la voix et des défauts del'ouie chez les enfants enTchécoslovaquie. Langage et Thomme, Brux. No. 7(1968).

Sprachstörungen bei Kindern; 3. ü. Auflage, p. 423 (VEB Verlag Volk und Gesundheit, Berlin 1969). 
Beziehungen zwischen Störungen der Vestibulozerebellarfunktion und verspätetem Sprachbeginn. Mschr. Ohrenheilk. Laryng. Rhinol., Heft 3, p. 103 (1969).

Relations motorics of speech and general motoric ability in clutterers. Folia phoniat. 22: 376380(1970). 